\title{
EFECTOS A CORTO PLAZO DE LA CONTAMINACIÓN ATMOSFÉRICA SOBRE LA MORTALIDAD: RESULTADOS DEL PROYECTO EMECAM EN EL MUNICIPIO DE MADRID, 1992-1995*
}

\author{
Iñaki Galán Labaca, Emiliano Aránguez Ruiz, Ana Gandarillas Grande, José María Ordóñez \\ Iriarte y Nuria Aragonés Sanz.
}

Dirección General de Salud Pública. Consejería de Sanidad y Servicios Sociales. Comunidad de Madrid.

$\left({ }^{*}\right)$ Este trabajo cuenta con una beca del Fondo de Investigaciones Sanitarias (expediente núm 97/0051-05).

\section{RESUMEN}

Fundamento: A pesar de los cambios producidos en las fuentes de emisión, los niveles de partículas, $\mathrm{SO}_{2}$ y y $\mathrm{CO}$ continúan siendo importantes en el municipio de Madrid. Por otro lado, contaminantes fotoquímicos, como el $\mathrm{NO}_{2} \mathrm{y} \mathrm{O}_{3}$, están adquiriendo una importancia creciente debido al incremento del tráfico rodado y los elevados niveles de insolación que tiene esta ciudad. El objetivo de este trabajo es establecer la relación a corto plazo entre los principales contaminantes y la mortalidad diaria en el municipio de Madrid, durante el pcriodo de 1992 a 1995, utilizando el protocolo estandarizado del proyecto EMECAM (Estudio Multicéntrico Español de Contaminación Atmosférica y Mortalidad).

Métodos: Las variaciones diarias de la mortalidad por todas las causas, menos las externas, en todas las edades y en las personas de 70 años y más, así como las del aparato circulatorio y respiratorio, se asocian con las variaciones diarias de partículas $\left(\mathrm{PM}_{10}\right), \mathrm{SO}_{2}, \mathrm{NO}_{2}, \mathrm{CO}$ y O 3 , mediante modelos de Poisson autorregresivos. Se controlan las variables estacionalidad, tendencia, temperatura, humedad relativa, gripe, día de la semana, y días festivos e inusuales.

Resultados: Se observan asociaciones positivas estadísticamente significativas del $\mathrm{SO}_{2}$ con todas las series de mortalidad analizadas, del $\mathrm{CO}$ con la mortalidad en las personas mayores de 69 años así como con la cardiovascular y respiratoria, y de las partículas con la mortalidad cardiovascular. El NO 2 también se relaciona de forma estadísticamente significativa con la mortalidad cardiovascular. Estos efectos son inmediatos, es decir, ocurren con los contaminantes del mismo dia. No se observaron asociaciones positivas significativas con $\mathrm{el} \mathrm{O}_{3}$.

Conclusiones: Estos resultados sugieren que, para un espectro amplio de contaminantes principales, los niveles actuales de contaminación atmosférica en el municipio de Madrid se asocian con un incremento de la mortalidad.

Palabras clave: Contaminación atmosférica. Mortalidad. Proyecto EMECAM. Regresión de Poisson.

Correspondencia:

Iñaki Galán

Servicio de Epidemiología.

O'Donnell, 52.

28009 Madrid.

Correo electrónico: inaki.galan@comadrid.es

ABSTRACT

\section{Short-term Effects of Air Pollution on the Mortality. Results of the EMECAM Project in the Municipality of Madrid, 1992-1995}

Background: Despite the changes which have taken place in the sources of emissions, the levels of particles, $\mathrm{SO}_{2}$ and $\mathrm{CO}$ continue to be high in the municipality of Madrid. Apart from this, photochemical pollutants, such as $\mathrm{NO}_{2}$ and $\mathrm{O}_{3}$ are taking on growing importance due to the increased number of cars and trucks on the road and the major degrees of sunlight in this city. The objective of this article is to set out the short-term relationship between the major pollutants and the daily death rate in the city of Madrid for the 1992-1995 period, using the standardized procedure of the EMECAM Projects (Spanish Multicenter Study of Air Pollution and Death Rate).

Methods: The daily fluctuations in the death rate for all causes except external ones for all ages and for those individuals over age 69 , in addition to those of the circulatory system and respiratory apparatus are related to the daily fluctuations in particles $\left(\mathrm{PM}_{10}\right), \mathrm{SO}_{2}, \mathrm{NO}_{2}, \mathrm{CO}$ and $\mathrm{O}_{3}$, by means of autoregressive Poisson regression models. The seasonality, tendency, temperature, relative humidity, flu, day of the week, holidays and events out of the ordinary are controlled.

Results: Statistically significant positive relationships were found to exist between $\mathrm{SO}_{2}$ and all of the death rate series analyzed, between $\mathrm{CO}$ and the death rate of individuals over age 69 , as well as with cardiovascular and respiratory deaths and of the particles to the death rate as the result of cardiovascular disease. A statistically significant relationship was also found to exist between $\mathrm{NO}_{2}$ and the cardiovascular death rate. These impact are immediate, that is to say, they occur with the pollutants of the same day. No significant positive relationships were found to exist for $\mathrm{O}_{3}$.

Conclusions: Thesc findings suggest that, for a broad spectrum of major pollutants, the current levels of air pollution in Madrid are related to a rise in the death rate.

Key words: Air pollution. Mortality. EMECAM Project. Poisson regression. 


\section{INTRODUCCIÓN}

La relación entre diversos indicadores de salud y las variaciones diarias de contaminación atmosférica ha sido estudiada en diversas ciudades de Europa y Estados Unidos ${ }^{1-6}$, encontrándose asociaciones con niveles de contaminación considerados bajos o inferiores a los niveles guía propuestos por la OMS en $1987^{7}$. De la misma forma, estudios previos realizados en el municipio de Madrid $^{8}$ han puesto en evidencia esta relación.

El municipio de Madrid es un importante núcleo de actividad que constituye el centro de gravedad de una densa área metropolitana que genera intensos flujos de transporte de mercancías y personas. De ello se derivan las principales fuentes de emisión de contaminantes que son, por orden de importancia, el tráfico, las calderas de calefacción $y$ agua caliente sanitaria $y$, en menor medida, la industria ${ }^{y}$. Madrid está a la cabeza de las provincias españolas en cuanto a parque automovilístico, con 586 vehículos a motor por cada mil habitantes ${ }^{10} \mathrm{y}$, dada su antigüedad, menos de la mitad de los automóviles dispone de catalizador. Por otro lado, la producción de calor para consumo doméstico está sufriendo una paulatina sustitución del carbón por gas natural o electricidad, con el consiguiente descenso de emisión de $\mathrm{SO}_{2}$. En nuestro entorno geográfico se presentan unas condiciones climatológicas directamente relacionadas con los niveles de inmisión, como es la frecuencia de situaciones anticiclónicas en verano y en invierno, las cuales dificultan la dispersión de contaminantes. En invierno, además, las condiciones de estabilidad atmosférica, determinadas por la influencia de las altas presiones continentales, se acentúan por la también frecuente inversión térmica que impide los movimientos verticales de las masas de aire $^{11}$. En verano, la alta insolación junto con las fuertes emisiones de NO y compuestos orgánicos volátiles, determinan la presencia de altos niveles de $\mathrm{O}_{3}$ troposférico. Actualmente, los niveles de contaminación de $\mathrm{NO}_{2}$ y $\mathrm{SO}_{2}$ del municipio de Madrid se sitúan en el rango medio alto respecto al de otras ciudades españolas participantes en el proyecto $E M E C A M$.

El municipio de Madrid presenta características interesantes para su participación en el proyecto EMECAM. Es la ciudad con mayor número de habitantes y dispone de una completa red de estaciones captadoras que mide una amplia variedad de contaminantes. Por otra parte, el estudio EMECAM ofrece la oportunidad de aplicar en nuestra área geográfica una metodología estandarizada en un período muy reciente, desde 1992 a 1995. El inicio en 1992 está motivado porque a partir de esta fecha la red de estaciones captadoras se amplía y se comienza a generalizar el registro de partículas de tamaño inferior a $10 \mu \mathrm{m}\left(\mathrm{PM}_{10}\right)$.

El objetivo de este trabajo es analizar la relación a corto plazo entre la contaminación atmosférica y la mortalidad diaria en el municipio de Madrid durante los años 1992 a 1995, siguiendo el protocolo estandarizado del proyecto EMECAM.

\section{METODOLOGÍA}

La metodología empleada es la desarrollada por el proyecto. Las variaciones diarias de mortalidad se relacionan con las variaciones diarias de contaminación cuando se estudian usando modelos autorregresivos de Poisson. A continuación se describen las características de las variables utilizadas en el estudio.

\section{Población y área de estudio}

La población media del municipio de Madrid para el período 1992-1995 (interpolación lineal entre el censo de 1991 y el padrón de 1996) es de 2.940 .896 habitantes, distribuidos en una superficie de $605,8 \mathrm{Km}^{2}$, lo que equivale a una densidad de 4.964 habitantes por $\mathrm{Km}^{2}$. 


\section{Mortalidad}

El número de defunciones registradas en el municipio de Madrid de personas residentes en el mismo para los años 1992 a 1995, se obtuvieron del Registro de Mortalidad de la Comunidad de Madrid. Se ha estudiado la mortalidad por todas las causas, menos las externas, en todas las edades y en personas de 70 y más años (CIE 9a 001-799), la mortalidad por enfermedades del aparato circulatorio (CIE 9 390-459), y la mortalidad por enfermedades del aparato respiratorio (CIE $\left.9^{a} 460-519\right)$.

\section{Datos de contaminación atmosférica}

El municipio de Madrid cuenta con una red automática denominada Red de Control de Contaminación Atmosférica ${ }^{13}$, compuesta por un total de 24 estaciones remotas dispersas a lo largo de todo el municipio. Esta red fue contrastada por el centro de la Unión Europea de Ispra en el año $1991^{14}$.

Los contaminantes y métodos analíticos utilizados son los siguientes: las partículas se han medido como $\mathrm{PM}_{10}$ promedio diarios (atenuación de la radiación $\beta$ ); el $\mathrm{SO}_{2}$ promedio diario y máximo horario (absorción de la fluorescencia ultravioleta); el $\mathrm{NO}_{2}$ promedio diario y máximo horario (quimiluminiscencia); el CO promedio diario (absorción por infrarrojo no dispersivo); el $\mathrm{O}_{3}$ promedio de 8 horas (absorción ultravioleta).

Para el período de estudio se ha prescindido de la información registrada en diversas estaciones, debido a cambios de emplazamiento, pérdida de datos o períodos de la serie con datos dudosos o extraños. Asimismo, se han excluido los datos de una estación alejada del casco urbano. El rango mínimo de lecturas válidas para toda la serie para cada contaminante en las estaciones captadoras incluidas es del $97 \%$. Los valores perdidos fueron imputados a partir de los valores obtenidos en la regresión de cada una de ellas, utilizando los datos de las de- más estaciones como variables explicativas. El número de estaciones utilizadas para cada contaminante es el siguiente: partículas, 12 estaciones; $\mathrm{SO}_{2}, 16$ estaciones; $\mathrm{NO}_{2}$, 15 estaciones; $\mathrm{CO}, 12$ estaciones; $\mathrm{O}_{3}, 3$ estaciones.

\section{Variables meteorológicas}

Se han utilizado la temperatura media y la humedad relativa mcdia, obtenidas a partir de cuatro mediciones diarias $(0,7,13$ y 18 horas) en el observatorio meteorológico de Barajas, situado en la zona noreste del municipio. Este observatorio, además de representativo del clima de Madrid, ofrece una contrastada información de alta calidad.

\section{Otras variables}

Los datos sobre incidencia de gripe se han obtenido del registro del Sistema de Enfermedades de Declaración Obligatoria, con una cobertura de notificación, para el período de estudio, que oscila entre el $88,5 \%$ y $89,6 \%$. La distribución temporal de la incidencia de gripe a través de esta fuente de información coincide con la observada por otros registros ${ }^{15}$.

También se incluyeron las variables estacionalidad, año, tendencia, día de la semana, huelga general y huelga médica, así como los días festivos correspondientes.

\section{Análisis estadístico}

La construcción de los modelos de regresión se realizó para cada contaminante y causa de mortalidad según el protocolo del proyecto $E M E C A M^{12}$. Se estudió la asociación de cada contaminante con la mortalidad de forma cruda y mediante su transformación logarítmica, excepto para el $\mathrm{O}_{3}$, para el que también se probó una transformación cuadrática. En la selección del mejor modelo se utilizó una descripción gráfica, la mejora en el ajuste del coeficiente de determi- 
nación realizando una regresión lineal, y la mejora del ajuste en el modelo final de regresión de Poisson.

Los resultados se expresan como el riesgo relativo (RR) de mortalidad para un incremento de $10 \mu \mathrm{g} / \mathrm{m}^{3}$ a partir de la mediana en los niveles de cada contaminante, excepto para el $\mathrm{CO}$ que se expresan para un incremento de $1 \mathrm{mg} / \mathrm{m}^{3}$. También se presenta el RR para un incremento correspondiente a la diferencia entre los niveles del percentil 90 y los del percentil 10. En este trabajo se estudia la asociación de cada uno de los contaminantes sin ajustar por los demás.

\section{RESULTADOS}

En la tabla 1 y en la figura 1 se puede observar la distribución de los contaminantes estudiados. Como era de esperar, el patrón estacional de los contaminantes es invernal, especialmente para $\mathrm{SO}_{2}, \mathrm{CO}$ y partículas, al coincidir el incremento de emisión de las mismas con condiciones meteorológicas propicias para la formación de inversiones térmicas, que incrementan la concentración de contaminantes en la atmósfera ${ }^{11}$. Por el contrario, el $\mathrm{O}_{3}$ presenta un comportamiento inverso, con máximos niveles en verano, dado que es un contaminante secundario que necesita la actuación de la luz solar además de los contaminantes precursores ${ }^{16}$.

En la tabla 2 se presentan las correlaciones entre los contaminantes y variables meteorológicas, en la que aparece una alta correlación positiva entre las partículas, $\mathrm{CO}, \mathrm{SO}_{2}, \mathrm{y}$ $\mathrm{NO}_{2}$. El $\mathrm{O}_{3}$ se correlaciona negativamente con el resto de contaminantes de la seric. La temperatura media se correlaciona de forma positiva con $\mathrm{el}_{3}$ y negativa con el resto de contaminantes. La correlación entre las estaciones captadoras es bastante alta para cada uno de los contaminantes, siendo la más baja de 0,4147 para el promedio diario de $\mathrm{NO}_{2}, \mathrm{y}$ la más alta de 0,9467 para el CO.

A excepción de unos pocos días, la concentración atmosférica de los contaminantes se mantiene por debajo de los valores guía propuestos por la $\mathrm{OMS}^{5}$. En el caso del $\mathrm{NO}_{2}$, sólo 4 días de la serie de promedios diarios superan los $150 \mu \mathrm{g} / \mathrm{m}^{3}$ y 2 días para la máxima horaria superan los $400 \mu \mathrm{g} / \mathrm{m}^{3}$. La con-

Tabla 1

Estadísticos descriptivos de la mortalidad diaria, contaminantes, variables meteorológicas y gripe. Municipio de Madrid 1992-1995

\begin{tabular}{|c|c|c|c|c|}
\hline Variables & Media (s.d.) & Percentil 10 & Percentil 50 & Percentil 90 \\
\hline $\begin{array}{l}\text { Mortalidad todas las causas menos ex- } \\
\text { ternas }\end{array}$ & $60,8(11,1)$ & 47 & 60 & 75 \\
\hline $\begin{array}{l}\text { Mortalidad todas las causas menos ex- } \\
\text { ternas en personas } \geq 70 \text { años }\end{array}$ & $40,5(8,7)$ & 30 & 40 & 52 \\
\hline Mortalidad aparato circulatorio & $21,7(6,1)$ & 14 & 21 & 30 \\
\hline Mortalidad aparato respiratorio & $6,1(3,1)$ & 3 & 6 & 10 \\
\hline Particulas $\left(\mu \mathrm{g} / \mathrm{m}^{3}\right)$ & $37,8(17,7)$ & 22,8 & 32,8 & 76,3 \\
\hline $\mathrm{SO}_{2} 24$ horas $\left(\mu \mathrm{g} / \mathrm{m}^{3}\right)$ & $35,5(27,2)$ & 11,9 & 25,9 & 76,9 \\
\hline $\mathrm{SO}_{2} 1$ hora $\left(\mu \mathrm{g} / \mathrm{m}^{3}\right)$ & $77,9(64,5)$ & 23,6 & 56,3 & 169,8 \\
\hline $\mathrm{NO}_{2} 24$ horas $\left(\mu \mathrm{g} / \mathrm{m}^{3}\right)$ & $70,9(20,0)$ & 48,7 & 68,1 & 96,7 \\
\hline $\mathrm{NO}_{2} 1$ hora $\left(\mu \mathrm{g} / \mathrm{m}^{3}\right)$ & $122,9(44,6)$ & 79,3 & 112,2 & 180,0 \\
\hline $\mathrm{CO}\left(\mathrm{mg} / \mathrm{m}^{3}\right)$ & $2,1(1,1)$ & 1,0 & 1,9 & 3,7 \\
\hline $\mathrm{O}_{3}\left(\mu \mathrm{g} / \mathrm{m}^{3}\right)$ & $42,1(27,8)$ & 9,6 & 79,1 & 98,7 \\
\hline Temperatura media $\left({ }^{\circ} \mathrm{C}\right)$ & $14,4(7,7)$ & 4,9 & 13,7 & 25,8 \\
\hline Humedad relativa $(\%)$ & $61,8(16,7)$ & 40,6 & 60,1 & 85,1 \\
\hline Gripe (casos diarios) & $1.200(1.084,1)$ & 139 & 964 & 2.534 \\
\hline
\end{tabular}


Figura 1

Variaciones diarias de la contaminación atmosférica. Municipio de Madrid, 1992-95
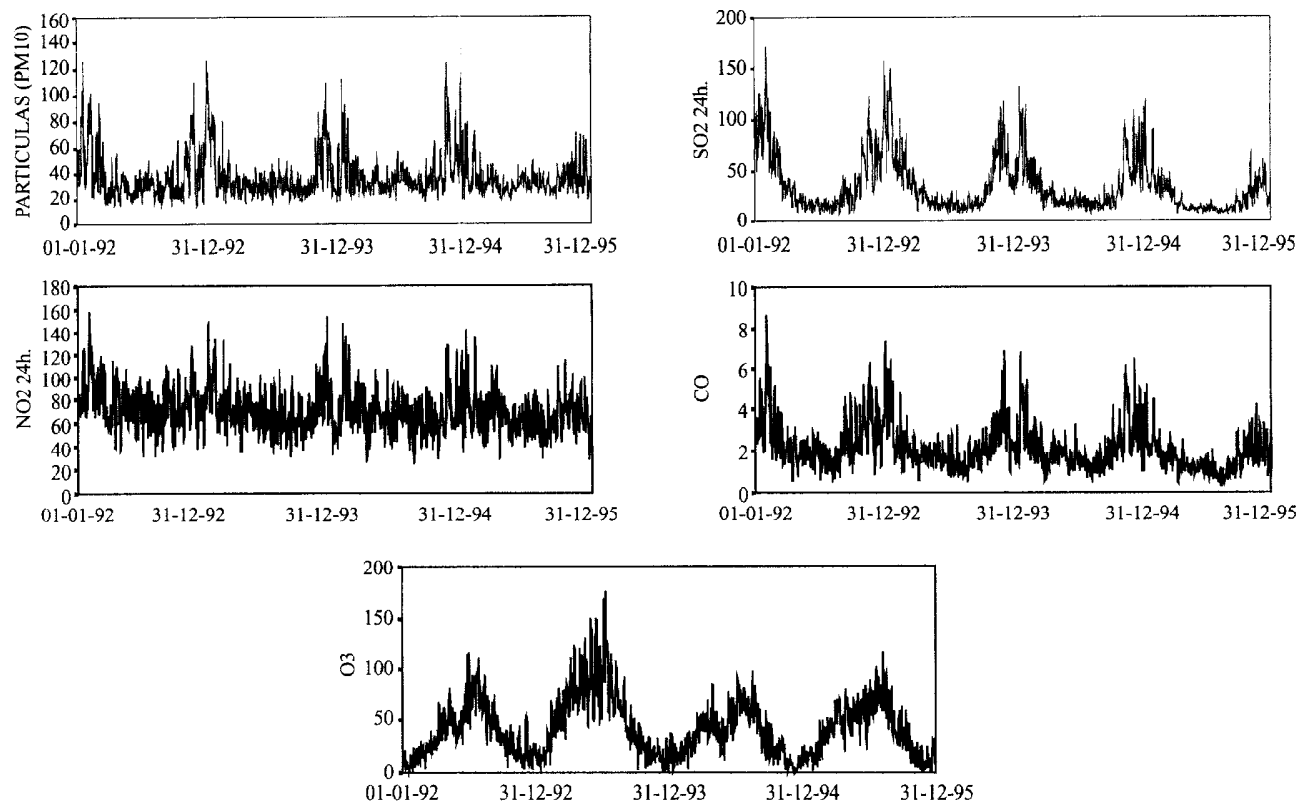

Tabla 2

Coeficientes de correlación de Pearson entre contaminantes y variables meteorológicas. Municipio de Madrid 1992-1995

\begin{tabular}{|c|c|c|c|c|c|c|c|c|c|}
\hline & Particulas & $\mathrm{SO}_{2} 24 \mathrm{~h}$ & $\mathrm{SO}_{2} \mathrm{Ih}$ & $\mathrm{NO}_{2} 24 \mathrm{~h}$ & $\mathrm{NO}_{2} \mathrm{Ih}$ & $\mathrm{CO}$ & $\mathrm{O}_{3}$ & Temp ${ }^{*}$ & Humedad * \\
\hline Partículas & 1 & & & & & & & & \\
\hline $\mathrm{SO}_{2} 24 \mathrm{~h}$ & 0,7821 & 1 & & & & & & & \\
\hline $\mathrm{SO}_{2}$ lh. & 0,7671 & 0,9740 & 1 & & & & & & \\
\hline $\mathrm{NO}_{2} 24 \mathrm{~h}$. & 0,7706 & 0,6991 & 0,7193 & 1 & & & & & \\
\hline $\mathrm{NO}_{2} 1 \mathrm{~h}$. & 0,7216 & 0,6457 & 0,7061 & 0,9088 & 1 & & & & \\
\hline $\mathrm{CO}$ & 0,8378 & 0,8816 & 0,8881 & 0,8132 & 0,7530 & 1 & & & \\
\hline $\mathrm{O}_{3}$ & $-0,3802$ & $-0,5593$ & $-0,4961$ & $-0,1693$ & $-0,0726$ & $-0,4922$ & 1 & & \\
\hline Temp. * & $-0,3584$ & $-0,6938$ & $-0,6179$ & $-0,2596$ & $-0,1496$ & $-0,5316$ & 0,6955 & 1 & \\
\hline Humedad * & 0,3609 & 0,4594 & 0,3738 & 0,1230 & 0,0084 & 0,4742 & $-0,6668$ & $-0,7034$ & 1 \\
\hline
\end{tabular}

* Temp = temperatura media: Humedad = humedad relativa.

centración máxima horaria de $\mathrm{SO}_{2}$ supera 6 días los $350 \mu \mathrm{g} / \mathrm{m}^{3}$ y el promedio de 8 horas de $\mathrm{O}_{3}$ supera 17 días $\operatorname{los} 120 \mu \mathrm{g} / \mathrm{m}^{3}$. I os valores de $\mathrm{SO}_{2}, \mathrm{NO}_{2}$ y CO, presentan en la serie una tendencia lineal descendente estadísticamente significativa. La tendencia descendente del $\mathrm{SO}_{2}$ parece ser consecuencia de la gradual sustitución de las calefacciones de carbón por las de gas natural y elec- tricidad. Por otro lado, la disminución de $\mathrm{NO}_{2}$ y CO, podría asociarse a la incorporación de catalizadores en los vehículos a motor, junto con la modernización del parque automovilístico.

En la tabla 1 se presenta un resumen de los estadísticos descriptivos de la mortalidad. La calidad de estos datos ha sido anali- 
zada previamente ${ }^{17}$. La media de fallecimientos diarios por todas las causas menos las externas y todas las edades, es de 60,8 , correspondiendo un $35,7 \%$ y un $10,1 \%$ a la mortalidad del aparato circulatorio y respiratorio respectivamente.

En la figura 2 se muestra el ajuste obtenido por el modelo al controlar la estacionalidad y la tendencia para la mortalidad diaria por todas las causas. Esta serie de mortalidad tiene un claro ciclo invernal que hace inducir su rclación con variables ambientales ${ }^{18}$. Asimismo, los picos de sobremortalidad estivales han sido asociados con la ocurrencia de altas temperaturas en esta época del año ${ }^{19}$. De hecho, la mayor mortalidad observada en nuestra serie ocurre en agosto de 1995, coincidiendo con temperaturas medias sostenidas de 30 y $31^{\circ}$, las máximas de todo el período.
En la tabla 3 se especifican los modelos basales correspondientes a las cuatro series de mortalidad estudiadas, sin incluir los términos autorregresivos. La mortalidad por todas las causas en personas de 70 y más años, es la que incluye mayor número de variables de control en el modelo, siendo el más parsimonioso el de la mortalidad respiratoria. Los residuos de los cuatro modelos basales, después de incluir de forma independiente los contaminantes, presentaban autocorrelaciones significativas que se solucionaron al incluir términos autorregresivos. Así, los modelos finales no presentan autocorrelación seriada y el análisis de los residuos no presenta un patrón evidente, a excepción de picos estivales relacionados con olas de calor. Por este motivo, pensamos que sería conveniente incluir esta variable en posteriores análisis, así como la de «vacaciones estiva-

Figura 2

Mortalidad observada y estimada después de ajustar por estacionalidad y tendencia. Municipio de Madrid, 1992-95

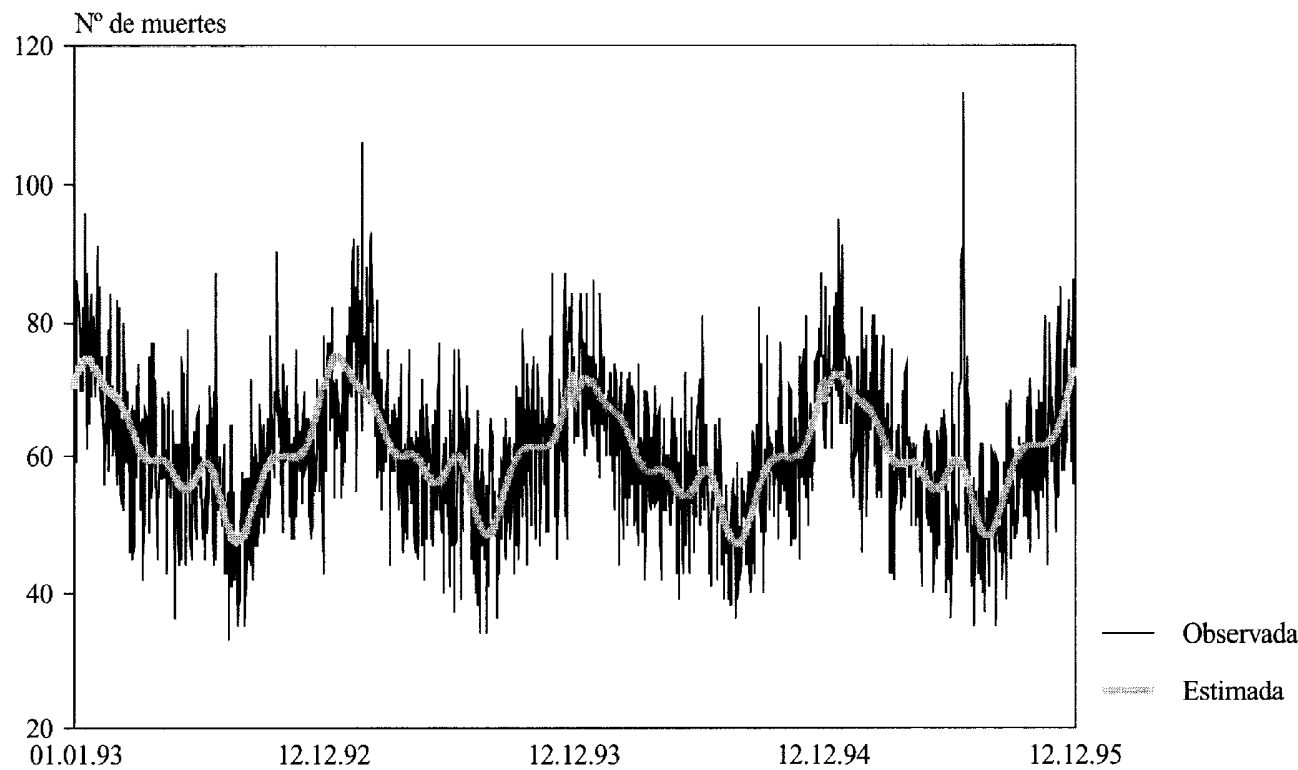


Tabla 3

Variables incluidas en el modelo basal para cada serie de mortalidad. Municipio de Madrid 1992-95

\begin{tabular}{|c|c|c|c|c|}
\hline \multirow{2}{*}{ Variables } & \multicolumn{4}{|c|}{ Mortalidad } \\
\hline & Todas menos externas & $\begin{array}{c}\text { Todas menos externas } \\
\geq 70 \text { años }\end{array}$ & Aparato circulatorio & Aparato respiratorio \\
\hline Estacionalidad & $\begin{array}{l}\text { Funciones senos-cosenos } \\
\text { de orden } 1 \text { al } 6\end{array}$ & $\begin{array}{l}\text { Funciones senos-cosenos } \\
\text { de orden } 1 \text { al } 6\end{array}$ & $\begin{array}{l}\text { Funciones senos-cosenos } \\
\text { de orden } 1 \text { al } 4\end{array}$ & $\begin{array}{l}\text { Funciones senos-cosenos } \\
\text { de orden } 1,3 \text { y } 4\end{array}$ \\
\hline Temperatura & $\begin{array}{l}\text { Lineal: } \operatorname{Ret}^{\#} 1,6 \\
\text { Cuadrática: Ret } 0,1\end{array}$ & $\begin{array}{l}\text { Lineal: Ret } 1,3,6 \\
\text { Cuadrática: Ret } 0,1,4\end{array}$ & $\begin{array}{l}\text { Lineal: Ret 1, } 6 \\
\text { Cuadrática: Ret } 1,6\end{array}$ & $\begin{array}{l}\text { Lineal: Ret } 1 \\
\text { Cuadrática: Ret } 1\end{array}$ \\
\hline Humedad & $\begin{array}{l}\text { Lineal: Ret } 3 \\
\text { Cuadrática: Ret } 0\end{array}$ & $\begin{array}{l}\text { Lineal: Ret } 2 \\
\text { Cuadrática: Ret } 0,2\end{array}$ & $\begin{array}{l}\text { Lineal: Ret } 0,3,7 \\
\text { Cuadrática: Ret } 0\end{array}$ & Lineal: Ret 6 \\
\hline Años* & 1993/94/95 (92 basal) & 1993/94/95 ( 92 basal) & 1993/94/95 (92 basal) & 1993/94/95 ( 92 basal) \\
\hline Tendencia & Cuadrática & - & - & Cuadrática \\
\hline Días de la Semana * & $\mathrm{M} / \mathrm{Mi} / \mathrm{J} / \mathrm{V} / \mathrm{S} / \mathrm{D}$ (L basal) & $\mathrm{M} / \mathrm{Mi} / \mathrm{J} / \mathrm{V} / \mathrm{S} / \mathrm{D}$ (L basal) & $\mathrm{M} / \mathrm{Mi} / \mathrm{J} / \mathrm{V} / \mathrm{S} / \mathrm{D}$ (L basal) & $\mathrm{M} / \mathrm{Mi} / \mathrm{J} / \mathrm{V} / \mathrm{S} / \mathrm{D}$ (L basal) \\
\hline Gripe & Ret 0 & Ret 0 & Ret 0 & Ret 5 \\
\hline Otras & Huelga médica & Huelga médica & 一 & - \\
\hline
\end{tabular}

\# Ret: Retardo

* Años y dias de la semana son variables «dummy» con múltiples categorias. Entre paréntesis la categoria de referencia

les» dada la gran disminución en nuestro área geográfica del número de personas expuestas en esta época del año.

En la tabla 4 se presentan los RR de cada contaminante y la mortalidad para todo el período, para un incremento del contaminante de $10 \mu \mathrm{g} / \mathrm{m}^{3}$ a partir de la mediana (excepto para el $\mathrm{CO}$ que es para un incremento de $\left.1 \mathrm{mg} / \mathrm{m}^{3}\right)$. En la figura 3 se exponen gráficamente estos resultados para incrementos equivalentes de la diferencia del percentil 90 y 10.

La relación del $\mathrm{SO}_{2}$ y CO con la mortalidad es de tipo logarítmico, mejorando el ajuste del modelo cuando se utiliza una transformación logarítmica de estos contaminantes. La relación funcional de tipo logarítmico entre el $\mathrm{SO}_{2}$ y la mortalidad, ha sido puesta de manifiesto en diferentes ciudades europeas ${ }^{20-22}$, así como en el munici-

Tabla 4

Riesgos relativos de la mortalidad por cada $10 \mu \mathrm{g} / \mathrm{m}^{3}$ de incremento del contaminante $\left(1 \mathrm{mg} / \mathrm{m}^{3}\right.$ para el CO) a partir de la mediana. Municipio de Madrid, 1992-95

\begin{tabular}{|c|c|c|c|c|c|c|c|c|}
\hline \multirow{3}{*}{ Contaminante } & \multicolumn{8}{|c|}{ Mortalidad } \\
\hline & \multicolumn{2}{|c|}{ Todas menos externas } & \multicolumn{2}{|c|}{$\begin{array}{l}\text { Todas menos externas } \\
\geq 70 \text { años }\end{array}$} & \multicolumn{2}{|c|}{ Aparato circulatorio } & \multicolumn{2}{|c|}{ Aparato respiratorio } \\
\hline & Retardo & $R R(I C 95 \%)$ & Retardo & $R R(I C 95 \%)$ & Retardo & $R R(I C 95 \%)$ & Retardo & $R R(I C 95 \%)$ \\
\hline Partículas & 4 & $0,9956(0,9912-1,0000)$ & 4 & $0,9950(0,9896-1,0004)$ & 0 & $1,0091(1.0015-1,0167)$ & 1 & $1,0080(0,9944-1,0218)$ \\
\hline Ln $\mathrm{SO}_{2} 24 \mathrm{~h} . \#$ & 0 & $1,0087(1,0011-1,0163)$ & 0 & $1,0116(1,0023-1,0210)$ & 0 & $1,0171(1,0050-1,0293)$ & 0 & $1,0276(1,0056-1,0500)$ \\
\hline In $\mathrm{SO}_{2} \mathrm{lh}$ \# & 0 & $1,0027(0,9999-1,0055)$ & 0 & $1,0045(1,0010-1,0080)$ & 0 & $1,0066(1,0021-1,0111)$ & 0 & $1,0110(1,0027-1,0193)$ \\
\hline $\mathrm{NO}_{2} 24 \mathrm{~h}$. & 3 & $0,9964(0,9926-1,0003)$ & 0 & $1,0039(0,9991-1,0086)$ & 0 & $1,0081(1,0018-1,0144)$ & 0 & $1,0107(0,9992-1,0222)$ \\
\hline $\mathrm{NO}_{2}=1 \mathrm{~h}$. & 3 & $0,9988(0,9971 \cdots 1,0004)$ & 0 & $1,0014(0,9994-1,0034)$ & 0 & $1,0029(1,0003-1,0056)$ & 0 & $1,0035(0,9987-1,0082)$ \\
\hline $\operatorname{Ln} \mathrm{CO} \#$ & 0 & $1,0081(0,9995-1,0168)$ & 0 & $1,0123(1,0017-1,0230)$ & 0 & $1,0199(1,0061-1,0340)$ & 0 & $1,0319(1,0064-1,0581)$ \\
\hline $\mathrm{O}_{3}$ & 4 & $1,0033(0,9987-1,0079)$ & 1 & $1,0051(0,9993-1,0109)$ & 1 & $0,9919(0,9841-0,9998)$ & 5 & $0,9911(0,9765-1,0058)$ \\
\hline
\end{tabular}

\# Transformación logaritmica del contaminantc. 
Riesgos relativos de la mortalidad para una diferencia del contaminante del percentil 90 y 10 . Municipio de Madrid, 1992-95

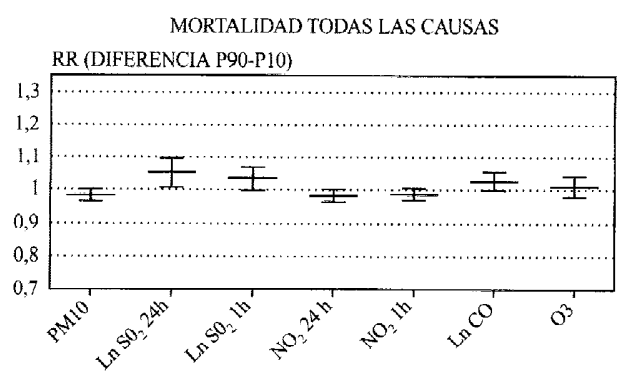

MORTALIDAD AP. CIRCULATORIO RR (DIFERENCIA P90-P10)

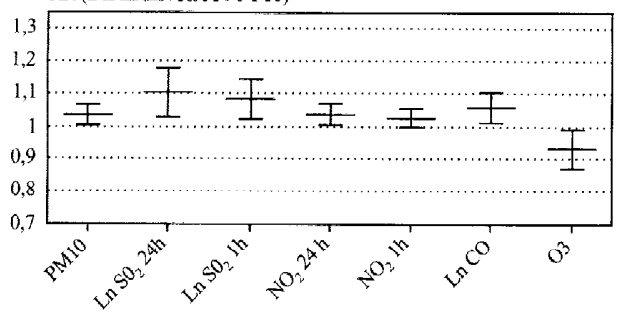

pio de Madrid ${ }^{8}$. A pesar de que la relación del $\mathrm{CO}$ y la mortalidad ha sido analizada con menor frecuencia, también se ha observado esta relación ${ }^{23}$. Sin embargo, no se mejora el ajuste después de realizar una transformación logarítmica y cuadrática del $\mathrm{O}_{3}$, por lo que se introduce como lineal.

$\mathrm{El} \mathrm{SO}$ es el contaminante que se asocia más claramente con la mortalidad, siendo esta relación ligeramente superior para el promedio diario que para la máxima horaria. La asociación de mayor magnitud del promedio diario de $\mathrm{SO}_{2}$ se presenta con la mortalidad respiratoria y cardiovascular, donde se alcanzan incrementos del $2,8 \%$ y $1,7 \%$, respectivamente, al aumentar este contaminante $10 \mu \mathrm{g} / \mathrm{m}^{3}$ a partir de la mediana. Este efecto es del $17 \%$ y $10 \%$ para una diferencia del percentil 90 y 10. La asociación del $\mathrm{SO}_{2}$ con la mortalidad se produce para el contaminante del mismo día.

El CO es, después del $\mathrm{SO}_{2}$, el contaminante con mayores efectos en la mortalidad.

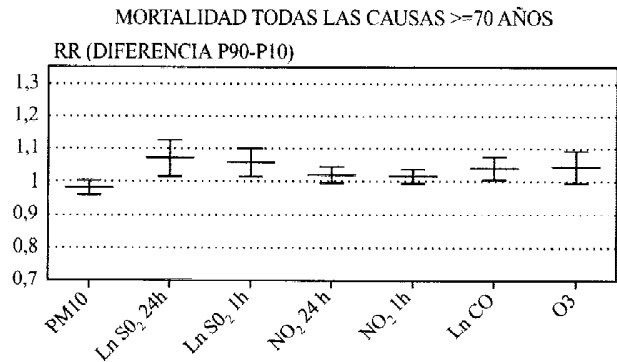

MORTALIDAD AP. RESPIRATORIO RR (DIFERENCIA P90-P10)

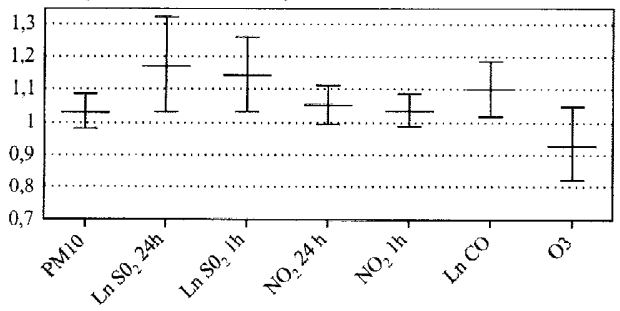

En concreto, se asocia de forma estadísticamente significativa con la mortalidad por todas las causas en las personas de 70 y más años, así como con la mortalidad de causa cardiovascular y respiratoria, estando en el límite de la significación con la mortalidad por todas las causas en todas las edades. El incremento de la mortalidad respiratoria es de un $3,2 \%$ para un incremento del $\mathrm{CO}$ de $1 \mathrm{mg} / \mathrm{m}^{3}$ a partir de la mediana, y de un $2 \%$ para las circulatorias. Al igual que para el $\mathrm{SO}_{2}$, su asociación se establece con el contaminante del mismo día.

Tanto el promedio diario de $\mathrm{NO}_{2}$ como la máxima horaria del mismo día, presentan una asociación estadísticamente significativa con la mortalidad cardiovascular, la cual se incrementa en un 3-4\% para diferencias del percentil 90 y 10. Su asociación con la mortalidad respiratoria y la de todas las causas en personas de 70 y más años está rozando la significación. El retardo 3 del $\mathrm{NO}_{2}$ presenta una relación negativa con la mortalidad por todas las causas en todas las edades, 
sin embargo, el $\mathrm{NO}_{2}$ del mismo día tiene una asociación positiva con un coeficiente de similar magnitud.

No se aprecia una relación entre las partículas y la mortalidad por todas las causas, pero aparece una asociación estadísticamente significativa del contaminante del mismo día con la mortalidad del aparato circulatorio, que se traduce en un incremento de un $3,5 \%$ para la diferencia del percentil 90 y el 10. También se manifiesta una asociación, aunque menos clara, en el retardo 1, con la mortalidad respiratoria.

$\mathrm{El} \mathrm{O}_{3}$ no presenta asociaciones positivas, a excepción de su relación en el retardo 1 con la mortalidad por todas las causas en las personas de 70 y más años.

\section{CONCLUSIONES}

Se ha encontrado asociación entre el $\mathrm{SO}_{2}$ y el $\mathrm{CO}$ con las cuatro series de mortalidad analizadas, mientras que las partículas $\left(\mathrm{PM}_{10}\right)$ se asocian con la mortalidad cardiovascular y, de forma menos clara, con la respiratoria.

$\mathrm{E} 1 \mathrm{NO}_{2}$ muestra una relación positiva estadísticamente significativa con la mortalidad cardiovascular, y no significativa con la mortalidad por todas las causas en personas de 70 y más años, y por causas respiratorias.

En relación con el $\mathrm{O}_{3}$, no hemos observado asociaciones positivas significativas. La relación del $\mathrm{O}_{3}$ con la mortalidad no es lineal sino curvilínea, apareciendo incrementos de mortalidad a bajas dosis de $\mathrm{O}_{3}$, una disminución de la mortalidad a medida que aumenta su concentración, y de nuevo un aumento de la mortalidad con altos niveles del contaminante. Esta relación hace que sea difícil su estudio con modelos paramétricos $y$, a pesar de que se probaron transformaciones de la variable (logarítmica y cuadrática), no se consiguió mejorar el ajuste.
Las asociaciones encontradas se producen de forma consistente con el contaminante del mismo día o en el retardo 1 para la relación de las partículas con la mortalidad respiratoria. Estos resultados indican que los efectos de las variables explicativas pueden ser inmediatos.

Este estudio tiene las limitaciones propias de los diseños de tipo ecológico, especialmente en la falta de precisión en la medida de las exposiciones. Asimismo, la pequeña magnitud de las asociaciones hace que la interpretación de los resultados deba realizarse con precaución, dado que variables no controladas en el modelo, o complejas relaciones entre contaminantes, pueden confundir sus resultados. Por todo ello debemos de ser cautos a la hora de sacar conclusiones de tipo causal.

Los resultados obtenidos presentan, para la mayor parte de los contaminantes, un importante grado de concordancia con los descritos por otros autores en situaciones de contaminación y para poblaciones no muy diferentes a las de nuestro entorno geográfico. En este contexto podemos concluir, contando con las precauciones señaladas anteriormente en la interpretación de los resultados, que las evidencias de este primer análisis de la asociación entre la contaminación atmosférica y la mortalidad en el municipio de Madrid en el marco del proyecto EMECAM, muestran que moderados niveles de contaminación atmosférica, en concreto de $\mathrm{SO}_{2}$, partículas, $\mathrm{CO}$ y $\mathrm{NO}_{2}$, incrementan de forma moderada la mortalidad, especialmente la debida a causas relacionadas con el aparato circulatorio y respiratorio.

\section{AGRADECIMIENTOS}

Agradecemos la información facilitada a Salvador Castromil y Francisco Moya, del Departamento de Control de Contaminación Atmosférica del Ayuntamiento de Madrid; a Santiago Jiménez Beltrán y Rafael Hernández, de la Subdirección General de Calidad 
Ambiental del Ministerio de Medio Ambiente; a Javier Mantero, del Instituto Nacional de Meteorología del Ministerio de Medio Ambiente; al Registro de Mortalidad de la Comunidad de Madrid, del Instituto de Estadística de la Consejería de Hacienda.

\section{BIBLIOGRAFÍA}

1. Scott JA. Fog and Deaths in London, Deccmber 1952. Public Health Rep 1953; 68:474-479.

2. Wichmann HE, Mueller W, Allhoff P, Beckmann M, Bocter N, Csicsaky MJ et al. Health effects during a smog episode in west Germany in 1985. Environ Health Perspect 1989;79:89-99.

3. Katsouyanni K, Zmirou D, Spix C, Sunyer J, Schouten JP, Pönka A et al. Short-term effects of air pollution on health: a European approach using epidemiological time-series data. Eur Resp J 1995;8:1030-1038.

4. Schwartz J. Air pollution and daily mortality: a review and meta Analysis. Environ Res 1994;64:36-52.

5. Sunyer J, Castellsagué J, Saez M, Tobias A, Antó JP. Air pollution and mortality in Barcelona. J Epidemiol Community Health 1996;50 Suppl 1:S76-S80.

6. Ballester F, Corella D, Pérez-Hoyos S, Hervás A. Air pollution and mortality in Valencia, Spain: a study using the APHEA methodology. J Epidemiol Community Health 1996;50:527-533.

7. World Health Organization. Air quality guidelines for Furope. WHO Regionals Publications, European Series núm 23. Copenhagen: WHO; 1987.

8. Díaz J, Alberdi JC, Montero JC, Mirón IJ. Asociación entre la contaminación atmosférica por dióxido de azufre y partículas totales en suspensión y la mortalidad diaria en la ciudad de Madrid (1986-1992). Gac Sanit 1998;12:207-215.

9. El medio Ambiente en la Comunidad de Madrid. Madrid: Consejería de Medio Ambiente y Desarrollo Regional; 1998 (en prensa).

10. Anuario estadístico general. Madrid: Dirección General de Tráfico. Ministerio del Interior; 1997.

11. López A, López J, Fernández F, Moreno A. El clima urbano. Teledetección de la isla de calor en Madrid. Madrid: Ministerio de Obras Públicas; 1993.
12. Protocolo EMECAM. Análisis del efecto a corto plazo de la contaminación atmosférica sobre la mortalidad. Rev Esp Salud Pública 1999; 73: 177-185.

13. Estaciones y redes de Vigilancia de la contaminación atmosférica en España. Descripción técnica y gráfica. Madrid: Ministerio de Obras Públicas, Transportes y Medio Ambiente; 1993.

14. Thibaut G, Lameloise PH, Le Moulec Y. Audit d'evaluation du reseau de surveillance de la qualité de l'air de la ville de Madrid vis a vis des Directives Européennes. París; 1991 (documento de trabajo).

15. Consejería de Sanidad y Servicios Sociales. Temporada de gripe 1995/1996 en la Comunidad de Madrid. Bol Epidemiol Comunidad de Madrid 1996;4:27-33.

16. McCurdy TR. Concentrations of ozone in the lower tropospheric (ambient air). En: McKee DJ, editor. Tropospheric ozone: human health and agricultural impacts. Boca Raton: Lewis Publishers;1994.p.19-37.

17. Indicadores de cobertura, calidad y depuración de la estadística. En: Estadísticas del movimiento natural de la población de la Comunidad de Madrid III. Defunciones 1990. Madrid: Consejería de Salud; 1993.p.57-61.

18. Alberdi JC, Díaz J. Modelización de la mortalidad diaria en la Comunidad Autónoma de Madrid (1986-1991). Gac Sanit 1997;11:9-15.

19. Montero JC, Mirón IJ, Díaz J, Alberdi JC. Influencia de variables atmosféricas sobre la mortalidad por enfermedades respiratorias y cardiovasculares en los mayores de 65 años de la Comunidad de Madrid. Gac Sanit 1997;11:164-170.

20. Schwartz J, Marcus A. Mortality and air pollution in London: a time series analysis. Am J Epidemiol 1990;131:185-194.

21. Dab W, Medina S, Quénel P, Le Moullec Y, Le Tertre A, Thelot B et al. Short term respiratory health effects of ambient air pollution: results of the APHEA project in Paris. J Epidemiol Comm Health 1996;50 (Suppl):S42-S46.

22. Vigotti MA, Rossi G, Bisanti L, Zanobetti A, Schwartz J. Short term effects of urban air pollution on respiratory health in Milan, Italy, 1980-89. J Epidemiol Comm Hcalth 1996;50 (Suppl):S71-S75.

23. Touloumi G, Spocock SJ, Katsouyanni K, Trichopoulos D. Short-term effects of air pollution on daily mortality in Athenas: a time-series analysis. Int J Epidemiol 1994;23:957-967. 